\title{
Pending Studies at Hospital Discharge
}

Molly A. Kantor, M.D., Kambria H. Evans, M.Ed., and Lisa Shieh, M.D., Ph.D.

Stanford University School of Medicine, Stanford, CA, USA.

J Gen Intern Med 30(8):1061

DOI: $10.1007 / \mathrm{s} 11606-015-3322-6$

(c) Society of General Internal Medicine 2015

$\mathrm{T}$ he Authors' Response-We thank Dr. Singh for highlighting a very important point. We agree that the prevalence of pending studies is a health systems problem with multifactorial origins, including shorter lengths of stay and increased handoffs. As Dr. Singh pointed out, and our study confirmed, physicians overestimate their awareness of pending studies. We showed that an EMR-based tool that automatically generated a list of pending studies from the EMR improved communication of pending studies via the discharge summary. We hope that the use of this tool will help change ordering behavior by allowing inpatient providers to become more aware of the volume of studies that are pending and for which they are responsible. In addition, at our institution, we are implementing quality improvement initiatives to try to change ordering behavior by displaying turnaround time and cost for some tests. Further work will need to be done in this area. In the meantime, the responsibility for pending studies falls to the inpatient provider (even if he or she disagrees with the test having been done in the first place), and communication to the primary care provider taking over care is essential. Our paper described a quality improvement intervention to help meet this need.

Conflict of interest: The authors declare that they do not have a conflict of interest.

Corresponding Author: Molly A. Kantor, M.D.; Stanford University School of Medicine, 300 Pasteur Drive, Lane 154, Stanford, CA 94305, USA (e-mail: mkantor2@stanford.edu). 